\title{
MECHATRONICS SYSTEMS
}

The term mechatronics refers to systems with special characteristics that can be achieved only as a whole, and cannot be realized through other means. Integrating mechanical and electronic components with control systems and informatics during design was introduced due to new performance requirements on system quality, its overall reliability, dynamics, low energy consumption, low weight, etc. The paper is focused on the basic information about mechatronics.

Keywords: mechatronics, robotics, integration, electortechnics, control

\section{Introduction}

Many systems in industry and other areas are designed for controlling mechanical movement. Although earlier systems were using only mechanical energy, most of the current mechatronics systems contain components for mechanical movement and parts that control it. The main drawback of earlier systems stems from the use of only simple laws of control, which in turn required system redesign for any control adjustments. Introducing electrical components into systems increased their flexibility. Additional significant improvement was enabled by microprocessors, which enabled to execute tasks by programs instead of purely by hardware. Combined, these improvements substantially increased the complexity of tasks that could be executed.

Integrating electrical, electronic and control systems with computers and mechanical systems established a new approach to design and use of manufacturing systems and other systems with mechanical movements.

Universal control systems comprise multiple components for: specification of control signals, implementation of control laws for static and dynamic events in the system, amplification of control commands, measurement of actual system state, and communication infrastructure. Control systems are usually implemented using computers, sensors for system state assessment, electric actuators for moving mechanical parts, and electric or optoelectric communication systems. The term mechatronics refers to such integrated systems with computer control of electric and mechanic subsystems.

\section{Mechatronics}

Growing demands for extended properties and capabilities of machines increase their complexity, which in turn requires improving the quality of these systems to the point when further improvements were not possible without integrating mechanical and electric systems during their design and use. Main directions of this approach, called mechatronics, were described in the 60-ties, and further developed by defining its principles in the 70-ties. Mechatronics relates to the start of modern robotics, and robots represent typical mechatronics systems. The main goal of this field is to design new generation of powerful and intelligent machines.

Although the term mechatronics was introduced in 1970, there is still discussion about its meaning. One line of thought defines mechatronics as nothing more than a good engineering design with systematic approach [2]. However, there are several definitions that clearly define goals of mechatronics as a scientific field, and there is plenty of research interest in the field $[3,4,5,6,7,8$, $9,10,11,12]$.

Currently, many fields and technologies synergistically integrate with computer science, resulting in increased use of autonomous, intelligent computerized control systems. This integration marks the beginning of "mechatronics age", which is characterized by fusion of diverse technologies and significant shortening of system design cycle.

In the past, system design was characterized by a sequential integration of individual design stages:

- Specifying mechanical components.

- Integrating sensors, actuators, and regulators.

- Adding control subsystems and programs.

In contrast, mechatronics follows an integrated and comprehensive approach to system design, where all design stages and components are integrated simultaneously. The result of such design approach should be a high-quality integrated system, which could not be achieved by sequential integration of individual design stages.

Mechatronics is the result of increased demands on automation, electronic and informatics approaches in engineering, and systems, in which mechanical movement needs to be precisely and flexibly controlled.

\footnotetext{
* Ladislav Jurišica

chairman of SOK committee for mechatronics, Faculty of Electrical Engineering and Information Technology, Slovak Technical University,

Bratislava, E-mail: ladislav.jurisica@stuba.sk
} 
Mechatronics integrates diverse techniques, approaches and principles including mechanics, electronics, control and information theory, and computer science to synthesize available subsystems to achieve required goals. Mechatronics system comprises nonseparable combination of mechanical and electronic components, which facilitate exchange of energy and information. Mechatronics is unique since it enables building systems that are qualitatively different from any previous designs [1]. The usual graphical representation of mechatornics is presented in Figures 1-3.

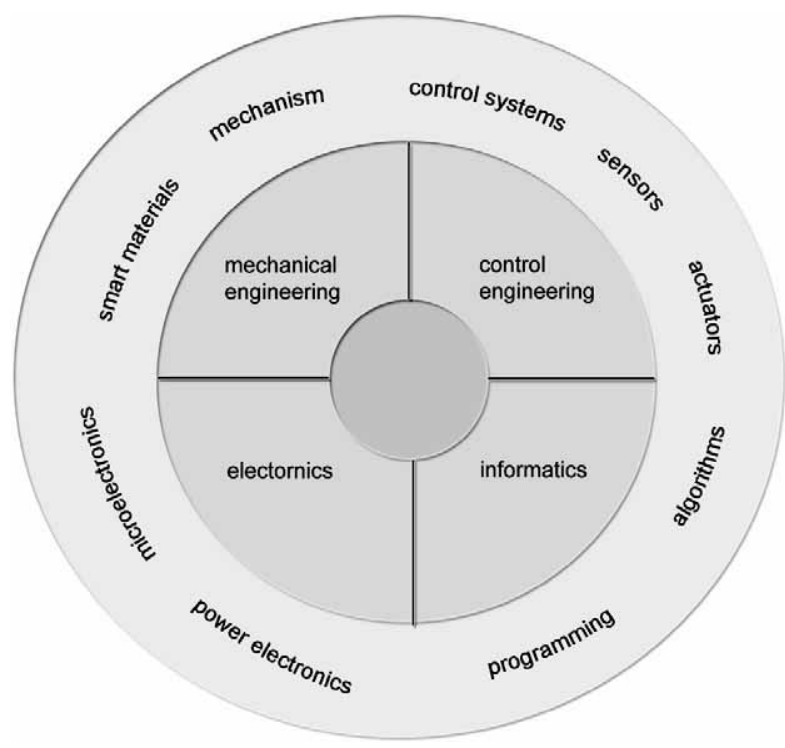

Fig.1. Mechatronics and its components

Technical systems with the software-oriented control may contain features, which were not attainable in the past, but may create challenges as well. Software is implemented in the computer system, but at the same time, it is a self-determining component, independent from the system implementation. Taking this into account, it is necessary to consider software quality and safety. Thus, new technologies require new methodology for software design, which has to be considered in the current definition of mechatronics.

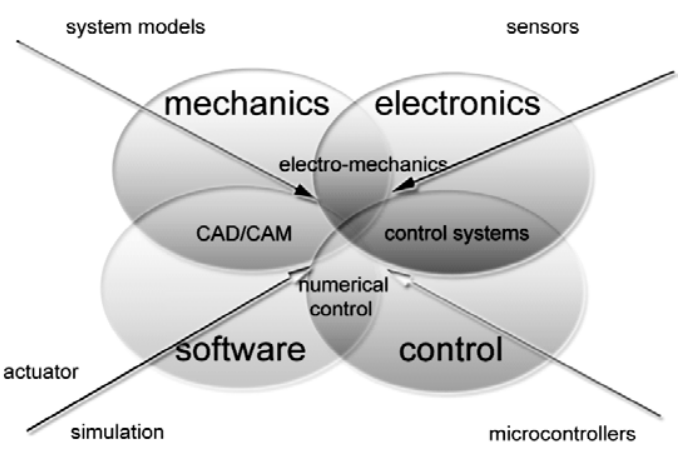

Fig. 2 Mechatronics as a combination of diverse technological and scientific disciplines
The classic definition characterizes mechatronics as a synergistic integration of precision machinery, electronics, computers, control systems, and system approach to design of processes and systems. More recent definitions include an important component - application of comprehensive decision-making process during the control of technological systems. The key element in mechatronics is the integration of all components in both the design and manufacturing processes. The important principle in mechatronics is the flexibility and ease of system and process control. The main goal is to create modules with "plug and play" features, and those having local autonomy.

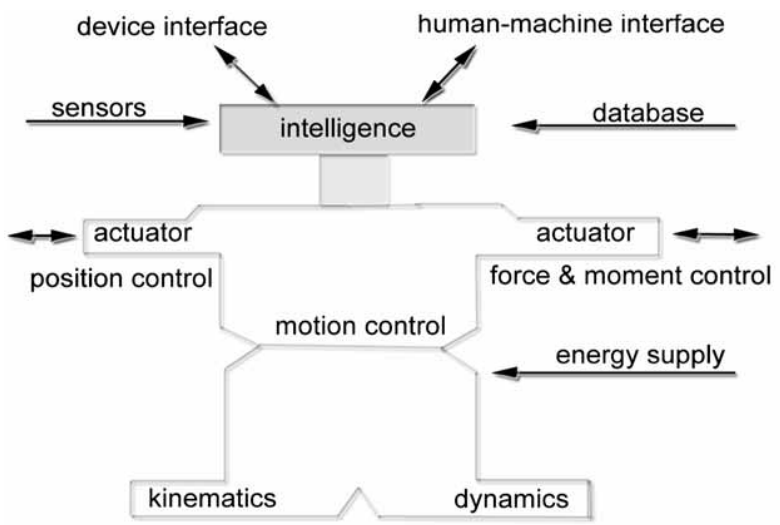

Fig. 3 Mechatronics systems and its integration with the environment

Mechatronics is a modern, interdisciplinary, technological and scientific field, which combines mechanics, electronics, informatics and cybernetics. Its goal is a synergistic integration of individual subsystems, in a comprehensive approach to design, manufacturing and use of machines, systems, components and products, in which main activity comprises mechanical principles.

Mechatronics focuses on computer-controlled electro-mechanical systems - mechatronics systems, their analysis and synthesis with the goal to achieve synergistic effect among individual subsystems, which are contained in the full system. Additional research focus also includes individual analysis and synthesis methods, design and manufacturing approaches, and experimental validation methods, since it is precisely those methods that are essential in achieving synergistic effect.

\section{Mechatronics Systems}

Mechatronics systems characterize a separate class of objects, which are qualitatively defined by the artificial systems with specific, intelligent behavior. This behavior is achieved by controlling generalized movements of individual parts of the system, and controlling energy interaction among individual diverse subsystems (including mechanical stiff and flexible matter, electromagnetic current, liquid, gas). For example, CNC machines, robots, technological automatic systems, mobile machines, transportation and manipulation systems, special machinery, consumer products, etc. 
A special class comprises micro-mechatronics systems - microelectro-mechanical systems (MEMSs).

One of the main requirements in mechatronics systems is to achieve intelligent properties for these systems. Intelligent systems could achieve the following:

- Sensing the environment and recognizing important and relevant information required for achieving the task.

- Anticipating changes in the environment considering the used model.

- Using information about the environment and expert knowledge for intelligent decision making.

- Planning the task execution required for achieving the given task and their adaptation to the actual state of the system environment.

- Communication and collaboration with other intelligent systems during problem solving.

- Learning from the past experience during solving the same or similar tasks.

- Adapting to a new environment.

The main goal of intelligent control is to achieve high system performance, high reliability, effective use, and automated fault response and recovery. Systems with sensors for monitoring the process are using decision support systems during considering imprecise information about parameters and changed signal, fuzzy control, artificial neural networks, learning, and other intelligent planning systems using artificial intelligence.

Mechatronics systems comprise three essential components: 1) energy components, 2) information-control component, and 3) mechanical subsystems.

Energy component comprises mechanisms, actuators and their connecting subsystems. The chief characteristic is the energy interaction among individual components.

Information-control component comprises sensors, communication and control subsystems (usually distributed), which process information and coordinated communication in mechatronics systems. Information system extension in mechatronics systems contains monitoring and diagnostics subsystems, visualization and automation systems for assessing quality of performed tasks, or the overall production quality. Information-control component is characterized by information processes and interactions.

Micro-mechatronics comprises integration of mechanical and electrical systems, where both subsystems are measured in microns. Micro-mechatronics requires combination of components such as microprocessors, microsensors and microactuators, with all functionality supported by intelligent computational systems. Additional essential features of such systems are their high functional reliability, low manufacturing cost, high reproducibility of functional characteristics, and low power requirements.

Design and manufacturing of microsystems require deep knowledge of physics principles of micro-components, and the ability to use powerful computer-aided design systems and simulators. The central assumption is the knowledge of microelectronics and micromanufacturing technologies. Micro-mechatronics is the future focus of mechatronics, in which existing composite materials will increasingly be complemented by novel materials, including biological.

\section{Conclusions}

Mechatronics is linked to increased demands on system performance. Gradually, electro-mechanical systems achieved intelligent properties. We can expect to achieve qualitatively new results only when comprehensive approach is used in design and synthesis of such systems.

Mechatronics systems comprise mainly electronic and opto-electronic components, for obvious reasons. It is precisely the advancement in micro-electronics and electronic materials that enabled realization of compact systems with sufficient mechanical power. Sophisticated structures, extensive sensor systems, powerful controlcomputer systems and actuators further enable new types of applications, including complex manufacturing processes.

Mechatronics approach to design is based on optimal system construction. This approach also leads to the use of smart materials in these systems.

\section{References}

[1] AUSLANDER, D. M.: What is Mechatronics?, IEEE/ASME Trans. on Mechatronics, 4/1996, pp. 5-9

[2] HEWIT, J. R., KING, T. G.: Mechatronic Design for Product Enhancement, IEEE/ASME Trans. on Mechatronics, 2/1996, pp. 111-119

[3] ACAR, M., PARKIN, R. M.: Engineering Education for Mechatronics, IEE Trans. on Ind. Electronics, 1/1996, pp. 106-112

[4]. FUKUDA, T., UEYAMA, T.: Self-Evolutionary Robotic System, J. of Robotics and Mechatronics, 2/1992, pp. 96-103

[5]. THIELEMANS, H.: HERDA - Heterogenous Distributed Real-time Architecture, Proc. Tampere Int. Conf. Machine Automation, 1994, pp. 627-639

[6] BOLTON, W.: Mechatronics. Electronic controlsystems in mechanical and electrical engineering, Pearson, Prentice Hall, 2003.

[7] DOVICA, M.: Components and modules in mini- and micro-mechanisms (in Slovak), Typo press, Košice 2002, ISBN 80-7099-878-4

[8] GMITERKO, A.: Mechatronics. Driving forces, characteristics and conceptual design of mechatronics systems (in Slovak), Košice, 2004, ISBN 80-8073-157-8

[9] http://sk.wikipedia.org/wiki/Mechatronika

[10] Mechatronics, AT\&P Journal Plus, 6/2005, ISSN 1336-5010

[11] Application of Artificial Intelligence, AT\&P Journal Plus, 7/2005, ISSN 1336-5010

[12] Mechatronics Systems, AT\&P Journal Plus, 1/2006, ISSN 1336-5010 